\title{
Priming reverse transcription with oligo(dT) does not yield representative samples of Mycobacterium tuberculosis cDNA
}

\author{
David L. Lakey, ${ }^{1,2,3}$ Yueru Zhang, ${ }^{1}$ Adel M. Talaat, ${ }^{4}$ Buka Samten, ${ }^{1}$ \\ Lucy E. DesJardin, ${ }^{5}$ Kathleen D. Eisenach, ${ }^{5}$ Stephen A. Johnston ${ }^{4}$ \\ and Peter F. Barnes $1,2,3$
}

Author for correspondence: Peter Barnes. Tel: +1 903877 7790. Fax: +1 9038775516. e-mail: peter.barnes@uthct.edu

\author{
1,2,3 Center for Pulmonary \\ and Infectious Disease \\ Control 1 , and \\ Departments of \\ Microbiology and \\ Immunology ${ }^{2}$ and \\ Medicine ${ }^{3}$, University of \\ Texas Health Center, \\ Tyler, TX 75708, USA \\ 4 Center for Biomedical \\ Inventions, University of \\ Texas Southwestern \\ Medical Center, Dallas, \\ TX 75390, USA \\ 5 Department of \\ Pathology, University of \\ Arkansas for Medical \\ Sciences and the Central \\ Arkansas Veterans \\ Health Care System, \\ Little Rock, AR 72205, \\ USA
}

\begin{abstract}
Several recent publications have suggested that oligo(dT) can prime reverse transcription of several mycobacterial mRNAs. To determine if this is the case for most Mycobacterium tuberculosis mRNA species, reverse transcription reactions of $M$. tuberculosis RNA were primed with oligo(dT) or with other primers that did not target polyadenylylated sequences, and the resultant CDNA product was evaluated. Priming with oligo(dT) yielded more CDNA than priming with an arbitrary primer for only 1 of 12 unrelated $M$. tuberculosis genes, as measured by competitive PCR. Priming with oligo(dT) yielded CDNA for only $\mathbf{3 0} \%$ of the genes primed for by $37 \mathrm{M}$. tuberculosis genome-directed oligonucleotides, as assessed by hybridization of CDNA with an M. tuberculosis microarray. These data demonstrate that priming of reverse transcription of mycobacterial mRNA with oligo(dT) does not yield representative samples of cDNA.
\end{abstract}

Keywords: mycobacteria, gene expression, transcription

\section{INTRODUCTION}

Tuberculosis causes a staggering burden of morbidity and mortality, and is responsible for an estimated 2 million deaths annually worldwide (Dye et al., 1999). The situation is worsened by the rising tide of drugresistant tuberculosis and the enormous population of susceptible hosts created by the pandemic of human immunodeficiency virus infection. More effective therapeutic and preventive measures for tuberculosis are desperately needed, and these hinge on an improved understanding of the basic biology of Mycobacterium tuberculosis.

The evaluation of gene expression by $M$. tuberculosis under different experimental conditions provides important insight into mycobacterial physiology, and these studies have been greatly facilitated by the availability of the genome sequences of M. tuberculosis (Cole et al.,

Abbreviation: gDNA, genomic DNA.
1998; http://www.tigr.org/tigr-scripts/CMR2/CMRGenomes.spl). However, analysis of mRNA expression by $M$. tuberculosis is difficult, because prokaryotic mRNA is not extensively polyadenylylated, and cannot be separated from total cellular RNA and DNA by binding to an oligonucleotide containing multiple thymidine nucleotides (dT). To overcome this problem, several groups have developed complex methods to study M. tuberculosis gene expression, such as selective capture of transcribed sequences and customized amplification libraries (Alland et al., 1998; Graham \& ClarkCurtiss, 1999).

In 1986, it was demonstrated that a poly(U) Sepharose column could isolate $0.8 \%$ of the total RNA isolated from Mycobacterium smegmatis, suggesting that a significant percentage of the mRNA produced by this organism was polyadenylylated (Katoch \& Cox, 1986). More recently, two publications showed that, after total RNA from M. tuberculosis, Mycobacterium bovis BCG, M. smegmatis and Mycobacterium vaccae was reverse transcribed with an oligo(dT) primer, several mycobacterial genes could be amplified by PCR from cDNA, 
supporting the concept that some mRNAs of $M$. tuberculosis and other mycobacteria are polyadenylylated (Adilakshmi et al., 2000; Rindi et al., 1998).

If all mycobacterial mRNAs are polyadenylylated, oligo(dT) can be used to isolate and prime reverse transcription of mRNA, permitting accurate quantification of mycobacterial mRNA expression. On the other hand, if only a limited number of mycobacterial mRNAs are polyadenylylated or if the extent of polyadenylylation of different mRNAs is variable, this strategy will not provide an accurate picture of mycobacterial gene expression. To determine if priming reverse transcription with oligo $(\mathrm{dT})$ yields representative samples of mycobacterial cDNA, we evaluated mRNA expression of multiple mycobacterial genes by competitive RT-PCR and by hybridization of mycobacterial cDNA to an M. tuberculosis microarray.

\section{METHODS}

Bacteria. M. tuberculosis strain H37Ra (no. 25177 from the American Type Culture Collection, Manassas, VA, USA) was grown to exponential phase for 5-7 days in Middlebrook $7 \mathrm{H} 9$ medium (Difco) and OADC (Remel) at $37^{\circ} \mathrm{C}$ and $5 \% \mathrm{CO}_{2}$, prior to isolation of total RNA.

RNA isolation. Mycobacteria were isolated by centrifugation and resuspended at $5 \times 10^{6}$ bacilli $\mathrm{ml}^{-1}$ in RNAzol B (TelTest), a chaotropic solution containing guanidine thiocyanate and phenol. The mycobacteria were disrupted by shaking with glass beads in an FP120 cell disrupter (Savant Instruments) at a setting of 6.5 for $23 \mathrm{~s}$, and total RNA was obtained by phenol/chloroform extraction, followed by DNase treatment.

Quantification of mRNA expression by competitive RT-PCR. We selected $12 \mathrm{M}$. tuberculosis genes that had the potential to contribute to virulence, based on published studies (Table 1). To quantify mRNA expression for these genes, we used the Omega 1.1 software program (Oxford Molecular) to design primers for each gene, based on the sequences available in the M. tuberculosis genome databases maintained by The Institute for Genomic Research and the Sanger Centre. The primer sequences are shown in Table 1 . The forward and reverse primers for $16 \mathrm{~S}$ rRNA were $5^{\prime}$-GGACTGAGATACGGCCCAGACT-3' and 5'-CGCGACAAACCACCTACGA-3', respectively. Those for $23 \mathrm{~S}$ rRNA were 5'-GAAACAGCCCAGATCGCC-3' and 5'-CCTACCCACACCCACCACA-3', respectively. In designing primers, the same criteria were used for each gene, thus minimizing differences in amplification efficiencies. Primers were $19-21 \mathrm{bp}$ in length, with a $\mathrm{G}+\mathrm{C}$ content of $60 \mathrm{~mol} \%$ and $T_{\mathrm{m}}$ of $60-65^{\circ} \mathrm{C}$, yielding amplicons of 150-220 bp. For all genes, competitors were constructed that were amplified by the same set of primers to yield amplicons 100-200 bp larger than those of the target sequences, using the MIMIIC system (Clontech Laboratory).

cDNA was prepared from total mycobacterial RNA by reverse transcription with MMLV reverse transcriptase (Gibco-BRL), and either an arbitrary oligonucleotide primer 5'-TTGAGGTAGGCGCGAAC-3' or a 15-mer oligo(dT) primer (both from Promega).

Tenfold serial dilutions of known amounts of MIMIIC DNA were added to $1 \mu \mathrm{l}$ of sample cDNA and amplified by PCR, using oligonucleotide primers specific for each gene. These preliminary experiments allowed a rough estimation of the amount of template cDNA. Serial twofold dilutions of MIMIIC DNA concentrations in the range of the estimated amount of template cDNA were then coamplified with sample cDNA by PCR. In this latter titration, the integrated density of the PCR product bands has a linear relationship to the amount of PCR product, and to the amount of template. PCR amplification was performed by running 28-35 cycles, each cycle consisting of denaturation at $94^{\circ} \mathrm{C}$ for $1 \mathrm{~min}$ and annealing/extension at $65^{\circ} \mathrm{C}$ for $2 \mathrm{~min}$. PCR products were subjected to electrophoresis and visualized by staining with ethidium bromide. To quantify PCR product, gels were photographed with a sPEEDLIGHT gel documentation system (B/T Scientific Technologies) and analysed with Quantity 1 software (Bio-Rad). This imaging and analysis system permits accurate comparison of the integrated density of the PCR product bands for target and MIMIIC DNA. By plotting the $\log$ of the ratio of integrated density of sample to MIMIIC PCR product against the log of the number of molecules of MIMIIC substrate DNA, the amount of substrate cDNA was determined, based on the point where the ratio of sample to MIMIIC PCR product was $1: 1$.

Table 1. Primers used for amplification of 12 mycobacterial genes

\begin{tabular}{|c|c|c|c|}
\hline Gene & Forward primer $\left(5^{\prime}-3^{\prime}\right)$ & Reverse primer $\left(5^{\prime}-3^{\prime}\right)$ & $\begin{array}{l}\text { Product size } \\
\text { (bp) }\end{array}$ \\
\hline Antigen $85 \mathrm{C}$ & ATCTGCTGGACGCCTTCAACG & ACTGCTTGCTGCCATCCTGC & 159 \\
\hline $\operatorname{aroB}$ & AGGTCGCCGTCGTGCATCAG & CCGATGCGGCCCAACACCTC & 165 \\
\hline efpA & GCCCTACGGGAAACCAACAA & CCACGATGACAAACGCGA & 184 \\
\hline$i d e R$ & CGATGTCATCGGGTTGCC & ACGCCAAGTTCCACCAGG & 159 \\
\hline katG & CCGCCATAGAGAAAGCAGCA & CCACCTACCAGCACCGTCA & 241 \\
\hline phoP & CGACGAGGAGACCCACGAA & TGCGGCGCAGATACGACA & 197 \\
\hline RNC & GCCGACAAGTCCAGCATTCT & AATCCAATCCCGCACCCA & 151 \\
\hline $\operatorname{sig} A$ & GTACAGGCCAGCCTCGAT & CCGATGACGACGAGGAGAT & 209 \\
\hline $\operatorname{sig} B$ & TTCATCGAGGACGCCGAA & GATCTGACGAACCCGCTCA & 207 \\
\hline $\operatorname{sir} R$ & TCTGAAGGTCATCTGGACCGCC & TCAACGTCACCGCGCCGTA & 164 \\
\hline $\operatorname{sod} A$ & CTTCACCACAGCAAGCACCA & GTCACCACCGTTAGGCGACA & 189 \\
\hline virS & GACCTCATCGAGCGCGAA & ACCGCTGACCCCACCATA & 180 \\
\hline
\end{tabular}


Microarray analysis. Total mycobacterial RNA was isolated as described above. The RNA was reverse transcribed in the presence of Cy3-dCTP (Amersham Pharmacia Biotech), 0·1 M DTT, $10 \mu \mathrm{M}$ dNTP except for $2.5 \mu \mathrm{M}$ dCTP (Gibco-BRL), Superscript II (Gibco-BRL) and its buffer, and either the oligo $(\mathrm{dT})$ or genome-directed primers, which were a mixture of 37 primers specifically designed to amplify all the ORFs in the M. tuberculosis genome (Talaat et al., 2000). Reverse transcription was performed at room temperature for $10 \mathrm{~min}$, followed by $42{ }^{\circ} \mathrm{C}$ for $2 \mathrm{~h}$. Unincorporated dye was removed by purifying the cDNA with QiaQuick purification kits (Qiagen) according to the manufacturer's protocol. Mycobacterial genomic DNA was nick-translated to generate DNA fragments of approximately $500 \mathrm{bp}(\mathrm{gDNA})$, and $2 \mu \mathrm{g}$ DNA was labelled with Cy5 fluorescent dye, according to the manufacturer's protocol (Promega). The Cy3-labelled cDNA was co-hybridized with Cy-5 labelled M. tuberculosis gDNA to an M. tuberculosis microarray that contained oligonucleotides (Operon Technologies) representing the 3924 ORFs present in the M. tuberculosis genome, as previously described (Kane et al., 2000; Call et al., 2001). Slides were allowed to hybridize overnight at $67^{\circ} \mathrm{C}$ before washing in low-stringency buffer $(1 \times \mathrm{SSC} / 0 \cdot 1 \% \mathrm{SDS})$ for $5 \mathrm{~min}$ at room temperature, followed by another $5 \mathrm{~min}$ wash in a highstringency buffer $(0 \cdot 1 \times$ SSC) and drying by centrifugation at 1000 r.p.m. for $5 \mathrm{~min}$. Dry slides were scanned at 10 micropixel intensity (GenePix4000, Axon Instruments).

The signal and background Cy3 and Cy5 fluorescence intensities were calculated for each DNA spot, using image analysis software (GenePix 3.0 Pro, Axon Instrument), to average the intensities of every pixel inside the target region. The intensity of each spot was calculated as the difference between mean signal intensity and mean local background intensity (segmentation method). The ratio of intensity for Cy3- to Cy5-labelled probes was determined for each DNA spot, reflecting the abundance of Cy3-labelled cDNA relative to Cy5-labelled gDNA. A ratio of 1 or greater was considered to represent a positive signal where the mRNA of a particular gene was expressed above the hybridization signal generated from its counterpart gDNA. All hybridizations were repeated two to four times before inclusion in the analysis.

\section{RESULTS}

\section{Oligo(dT) is not more effective than an arbitrary primer in initiating cDNA synthesis of $12 \mathrm{M}$. tuberculosis genes}

Previous studies have shown that oligo(dT) can prime cDNA synthesis for several mycobacterial genes (Adilakshmi et al., 2000; Rindi et al., 1998). We compared the efficiency of oligo(dT) and an arbitrary primer in initiating cDNA synthesis. If most mycobacterial mRNAs are polyadenylylated, the oligo(dT) primer should bind to most mRNA species much more efficiently than the arbitrary primer, resulting in a far greater yield of cDNA.

RNA was extracted from two separate cultures of $M$. tuberculosis, and each RNA sample was divided into equal aliquots. Each aliquot was reverse transcribed to cDNA, using either the oligo (dT) primer or the arbitrary primer. The resultant cDNA was amplified by com-
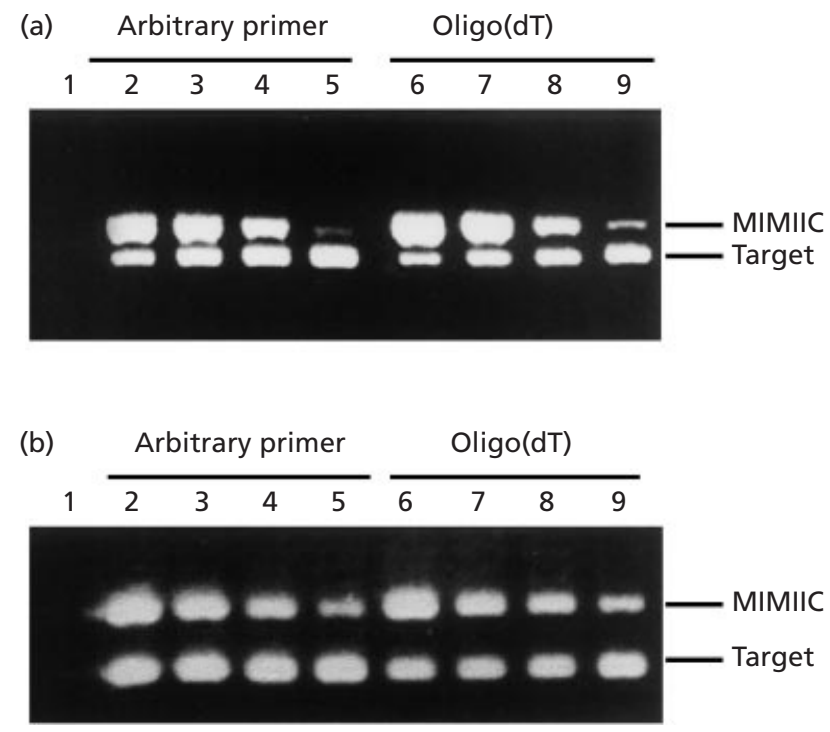

Fig. 1. Amplification of mycobacterial cDNA by competitive PCR, after priming reverse transcription of RNA with an arbitrary primer or with oligo(dT). (a) Results for amplification using primers for the sigA gene. Equal aliquots of an RNA preparation were reverse transcribed with an arbitrary primer (lanes 2-5) or with oligo(dT) (lanes 6-9), and amplified by competitive PCR. Lane 1, RNA template without reverse transcription; lanes 2-5, RNA template was reverse transcribed with an arbitrary primer, and MIMIIC competitors were added in 10-fold serial dilutions, beginning in lane 2; lanes 6-9, RNA template was reverse transcribed with oligo(dT), and MIMIIC competitors were added in 10-fold serial dilutions, beginning in lane 6. (b) Results for amplification using primers for the gene encoding antigen $85 \mathrm{C}$. Lane 1, RNA template without reverse transcription; lanes 2-5, RNA template was reverse transcribed with an arbitrary primer, and the MIMIIC competitors were added in twofold serial dilutions, beginning in lane 2; lanes 6-9, RNA template was reverse transcribed with oligo(dT), and the MIMIIC competitors were added in twofold serial dilutions, beginning in lane 6 .

petitive PCR, using primers for the $12 \mathrm{M}$. tuberculosis genes in Table 1. Serial dilutions of known quantities of MIMIIC DNA were added to template cDNA. Representative results are shown in Fig. 1 for $\operatorname{sig} A$ and for the gene encoding antigen $85 \mathrm{C}$.

Image analysis software was used to compare the integrated density of the PCR product bands for target and MIMIIC DNA and to calculate the amount of template cDNA for each gene produced by reverse transcription with the oligo(dT) primer or the arbitrary primer. These amounts were expressed as a ratio (Fig. 2 ). For eight genes, the amounts of cDNA obtained after priming with oligo(dT) were comparable to those obtained after priming with the arbitrary primer. For three genes (virS, sodA and katG), priming with oligo $(\mathrm{dT})$ yielded less cDNA than priming with the arbitrary primer (Fig. 2a). Only in the case of sirR did priming with oligo(dT) yield more cDNA (Fig. 2b). Therefore, for most of the genes tested, the amount of 

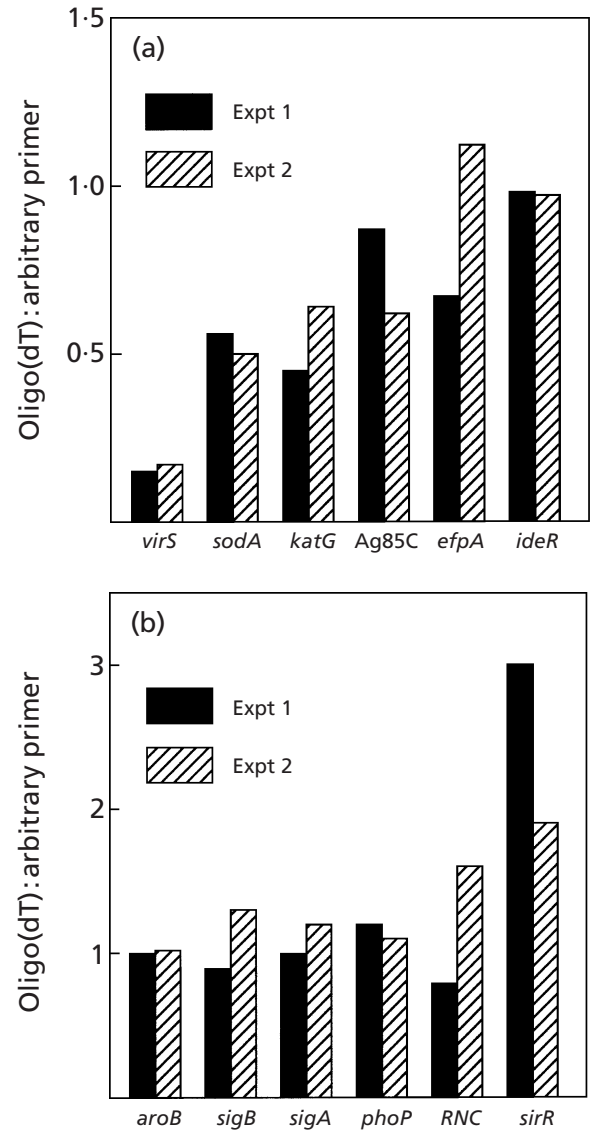

Fig. 2. Results of amplification of CDNA for $12 \mathrm{M}$. tuberculosis genes by competitive $P C R$, after priming reverse transcription of RNA with oligo(dT) or with an arbitrary primer. Equal aliquots of two RNA preparations were reverse transcribed with oligo(dT) or with an arbitrary primer, and amplified by PCR. The ratio of template cDNA after priming with oligo(dT) versus priming with an arbitrary primer is shown on the $y$-axis. Panels (a) and (b) each show results for six $M$. tuberculosis genes.

cDNA produced by priming with oligo(dT) was the same or less than that produced by priming with an arbitrary primer.

\section{Oligo(dT) primes reverse transcription of rRNA}

Recent studies documented polyadenylylation in approximately $10 \%$ of $23 \mathrm{~S}$ rRNA of Escherichia coli but in only $0.6 \%$ of $16 \mathrm{~S}$ rRNA (Mohanty \& Kushner, 2000). If these findings are similar for M. tuberculosis, and if reverse transcription with the oligo(dT) primer depends primarily on binding to polyadenylylated sequences, $16 \mathrm{~S}$ or $23 \mathrm{~S}$ rRNA should be reverse transcribed to a much lesser extent by oligo(dT) than by the arbitrary primer. However, when equal amounts of total M. tuberculosis RNA were reverse transcribed by oligo $(\mathrm{dT})$ or the arbitrary primer, the amounts of cDNA for the $16 \mathrm{~S}$ and $23 \mathrm{~S}$ rRNA were similar (Fig. 3).

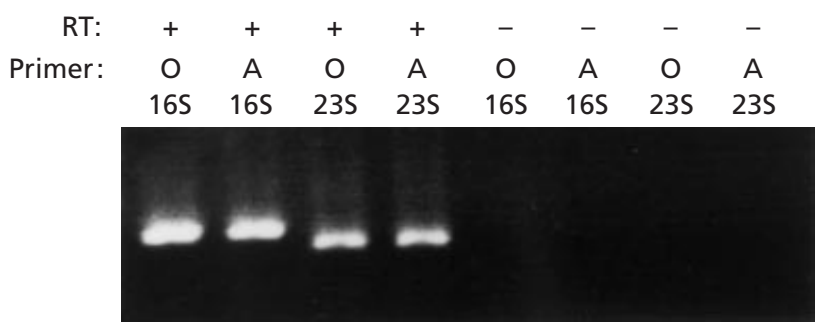

Fig. 3. Amplification of cDNA made from mycobacterial $16 \mathrm{~S}$ and $23 \mathrm{~S}$ rRNA, after priming reverse transcription with oligo(dT) or with an arbitrary primer. Equal aliquots of an RNA preparation were reverse transcribed with oligo(dT) or with an arbitrary primer, and amplified by PCR with primers for the CDNA for 16S rRNA or 23S rRNA (lanes 1-4). Aliquots of RNA were also amplified in the absence of reverse transcription (lanes 5-8). RT, reverse transcription; O, oligo(dT); A, arbitrary primer.

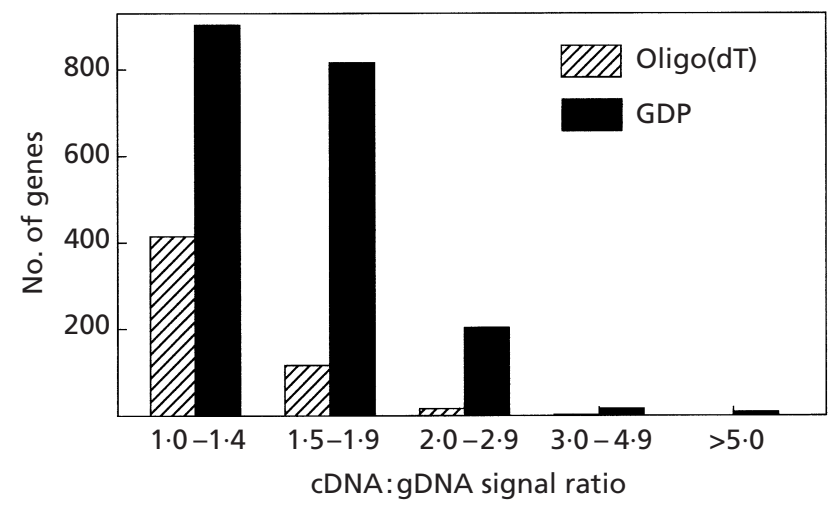

Fig. 4. Microarray analysis of mycobacterial CDNA, after priming reverse transcription of $M$. tuberculosis RNA with oligo(dT) or with genome-directed primers. The cDNA was hybridized to an $M$. tuberculosis microarray. The number of genes with cDNA/gDNA signal ratios of 1 or greater is shown.

\section{Microarray analysis demonstrates that a limited fraction of mycobacterial mRNAs is amplified by oligo(dT)-primed cDNA synthesis}

As an alternative means to evaluate the extent of polyadenylylation of $M$. tuberculosis mRNA, mycobacterial RNA was reverse transcribed with either the oligo $(\mathrm{dT})$ primer or the genome-directed primers. The resultant cDNA was hybridized to an M. tuberculosis microarray, and the efficiency of the two methods was compared by the signal ratios of cDNA/gDNA obtained by priming with oligo $(\mathrm{dT})$ or with genome-directed primers (Fig. 4). The same batch of labelled gDNA was used in both hybridizations to normalize for different hybridization efficiencies and different scanning settings. When total RNA was primed with oligo(dT), 550 (14\%) 
of the 3924 predicted mycobacterial ORFs had signal ratios $\geqslant 1$, the highest value being 3.9. Expression of 114 genes was detected by priming with oligo(dT) but not by priming with genome-directed primers. In contrast, when the same batch of RNA was primed with the genome-directed primers, 1847 (47\%) of the genes had signal ratios $\geqslant 1$, the highest ratio being $14 \cdot 5$. This included 1411 genes for which expression was not detected by priming with oligo(dT). Overall, more genes with higher signal intensity were detected by priming with the genome-directed primers than with oligo $(\mathrm{dT})$. If mycobacterial mRNA were extensively polyadenylylated, most ORFs would be more efficiently reverse transcribed with oligo $(\mathrm{dT})$. Therefore, these data suggest that the majority of M. tuberculosis mRNAs are not extensively polyadenylylated.

\section{DISCUSSION}

Our understanding of the biology of pathogenic organisms has been greatly enhanced by evaluation of mRNA expression under different conditions. Previous studies have shown that oligo $(\mathrm{dT})$ can prime cDNA synthesis for mRNA of the mycobacterial genes hsp65, entD, ent $C$ and trpE2 in M. bovis BCG, M. smegmatis and $M$. vaccae (Adilakshmi et al., 2000), as well as hsp65 and the gene encoding antigen $85 \mathrm{C}$ in M. tuberculosis and $M$. bovis BCG (Rindi et al., 1998). In this study, we used competitive PCR to show that the amount of cDNA produced by priming with oligo $(\mathrm{dT})$ was the same as or less than that produced by priming with an arbitrary oligonucleotide for 11 of $12 \mathrm{M}$. tuberculosis genes, including the gene encoding antigen 85C. Microarray analysis showed that genome-directed oligonucleotides primed cDNA synthesis for more M. tuberculosis genes than did oligo(dT). These data demonstrate that priming reverse transcription of $M$. tuberculosis RNA with oligo(dT) does not yield a representative sample of mycobacterial cDNA.

Although prokaryotic mRNA has traditionally been regarded as lacking $3^{\prime}$ polyadenylylated sequences, more recent studies have demonstrated that polyadenylylation occurs in a variety of bacteria, including $E$. coli and Bacillus species (Sarkar, 1996, 1997). Bacterial polyadenylylated tracts are 10-60 nucleotides long, significantly shorter than the 80-200 nucleotides found in eukaryotic cells. In addition, for any single mRNA species, only $2-50 \%$ of mRNA molecules are polyadenylylated, compared to $100 \%$ in the case of eukaryotes (Sarkar, 1997). Polyadenylylation is believed to be important for stability, maturation and translation of mRNA in eukaryotes. In contrast, a growing body of evidence suggests that polyadenylylation destabilizes certain bacterial mRNA species with stem-loop structures, contributing to their degradation (Sarkar, 1997).

Hybridization with an M. tuberculosis microarray revealed that oligo $(\mathrm{dT})$ primed cDNA synthesis for only 550 genes, whereas priming with genome-directed primers yielded hybridization signals for 1847 genes. It is possible that the number of mRNAs primed by oligo(dT) was underestimated because the large amount of rRNA may depress cDNA synthesis from mRNA templates. In addition, we only considered a significant amount of cDNA to have been synthesized if hybridization of cDNA to the microarray equalled that of an arbitrary amount of gDNA. This method reduces the number of non-specific signals, but it may underestimate the number of mRNAs for which oligo(dT) can prime reverse transcription.

Although the $3^{\prime}$ end of mycobacterial mRNA has not been sequenced, it has been hypothesized that polyadenylylation is present, because oligo(dT) can prime cDNA synthesis for several mycobacterial genes, which can then be amplified by non-competitive PCR (Adilakshmi et al., 2000; Rindi et al., 1998). Although we confirmed these results, we found that the quantities of cDNA synthesized after priming with oligo(dT) or with an arbitrary primer were very similar, arguing against the presence of extensively polyadenylylated mycobacterial mRNA, which would be more efficiently reverse transcribed by oligo $(\mathrm{dT})$.

The mechanism by which oligo $(\mathrm{dT})$ primes reverse transcription of mycobacterial mRNA is uncertain. We speculate that the mRNA is reverse transcribed by nonspecific binding of the oligo(dT) primer to the RNA template. When oligo $(\mathrm{dT})$ is used to prime reverse transcription of eukaryotic mRNA, selective binding to the mRNA polyadenylylated tail minimizes random binding to other segments of mRNA and rRNA. However, even under these conditions, rRNA is occasionally reverse transcribed. When oligo $(\mathrm{dT})$ is used to prime reverse transcription of mycobacterial mRNA, which is not extensively polyadenylylated, genes and rRNA with segments of several contiguous adenosines may function as the favoured binding sites for oligo(dT) at the relatively low temperature of $37^{\circ} \mathrm{C}$ used for reverse transcription.

Our results do not exclude the possibility that oligo(dT) primes reverse transcription of mycobacterial mRNA by specific binding to the polyadenylylated 3 ' ends, and that a variable percentage of $M$. tuberculosis mRNA species are polyadenylylated. This could explain the variable priming efficiency of oligo(dT) for different mycobacterial mRNAs, compared to a random primer (Fig. 2). In addition, microarray analysis revealed that oligo(dT) primed reverse transcription of 114 genes more efficiently than genome-directed primers, suggesting that these genes may be polyadenylylated. However, if variable degrees of limited polyadenylylation are present, reverse transcription with oligo(dT) cannot be used to obtain a representative assessment of mRNA expression under different conditions or by different $M$. tuberculosis strains. Such studies are best pursued by using a mixture of primers that are specifically designed to amplify all the ORFs in the M. tuberculosis genome (Talaat et al., 2000). Alternatively, because some mRNAs were amplified by oligo(dT) but not by the genome-directed primers, a combination of oligo $(\mathrm{dT})$ and genome-directed primers may yield the most com- 
prehensive collection of cDNAs. Others have used a combination of oligo(dT) and multiple arbitrary primers to perform mRNA differential display in two $M$. tuberculosis strains (Rindi et al., 1999).

In summary, using competitive PCR and microarray analysis, we found that priming of reverse transcription of mycobacterial mRNA species with oligo(dT) amplifies a limited fraction of mycobacterial mRNAs, and does not yield representative samples of cDNA.

\section{ACKNOWLEDGEMENTS}

This work was supported by grants from the National Institutes of Health (AI44935 and HL04298), and the Cain Endowment for Infectious Disease Research. Peter F. Barnes holds the Margaret E. Byers Cain Chair for Tuberculosis Research.

\section{REFERENCES}

Adilakshmi, T., Ayling, P. D. \& Ratledge, C. (2000). Polyadenylation in mycobacteria: evidence for oligo(dT)-primed cDNA synthesis. Microbiology 146, 633-638.

Alland, D., Kramnik, I., Weisbrod, T. R., Otsubo, L., Cerny, R., Miller, L. P., Jacobs, W. R., Jr \& Bloom, B. R. (1998). Identification of differentially expressed mRNA in prokaryotic organisms by customized amplified libraries (DECAL): the effect of isoniazid on gene expression in Mycobacterium tuberculosis. Proc Natl Acad Sci U S A 95, 13227-13232.

Call, D. R., Chandler, D. P. \& Brockman, F. (2001). Fabrication of DNA microarrays using unmodified oligonucleotide probes. Biotechniques 30, 368-372.

Cole, S. T., Brosch, R., Parkhill, J. \& 39 other authors (1998). Deciphering the biology of Mycobacterium tuberculosis from the complete genome sequence. Nature 393, 537-544.
Dye, C., Scheele, S., Dolin, P., Pathania, V. \& Raviglione, M. D. (1999). Global burden of tuberculosis - estimated incidence, prevalence, and mortality by country. JAMA ( $\mathrm{Am}$ Med Assoc) 282, 677-686.

Graham, J. E. \& Clark-Curtiss, J. E. (1999). Identification of Mycobacterium tuberculosis RNAs synthesized in response to phagocytosis by human macrophages by selective capture of transcribed sequences (SCOTS). Proc Natl Acad Sci USA 96, 11554-11559.

Kane, M. D., Jatkoe, T. A., Stumpt, C. R., Lu, J., Thomas, J. D. \& Madore, S. J. (2000). Assessment of the sensitivity and specificity of oligonucleotide (50mer). Nucleic Acids Res 28, 4552-4557.

Katoch, V. M. \& Cox, R. A. (1986). Step-wise isolation of RNA and DNA from mycobacteria. Int J Lepr 54, 409-415.

Mohanty, B. K. \& Kushner, S. R. (2000). Polynucleotide phosphorylase, Rnase II and Rnase E play different roles in the in vivo modulation of polyadenylation in Escherichia coli. Mol Microbiol 36, 982-994.

Rindi, L., Lari, N., Gil, M. G. \& Garzelli, C. (1998). Oligo(dT)primed synthesis of cDNA by reverse transcriptase in mycobacteria. Biochem Biophys Res Commun 248, 216-218.

Rindi, L., Lari, N. \& Garzelli, C. (1999). Search for genes potentially involved in Mycobacterium tuberculosis virulence by mRNA differential display. Biochem Biophys Res Commun 258, 94-101.

Sarkar, N. (1996). Polyadenylation of mRNA in bacteria. Microbiology 142, 3125-3133.

Sarkar, N. (1997). Polyadenylation of mRNA in prokaryotes. Annu Rev Biochem 66, 173-197.

Talaat, A. M., Hunter, P. \& Johnston, S. A. (2000). Genomedirected primers for selective labeling of bacterial transcripts for DNA microarray analysis. Nat Biotechnol 66, 679-682.

Received 9 October 2001; revised 17 April 2002; accepted 30 April 2002. 